\title{
AVALIAÇÃO DA SOBRECARGA EMOCIONAL DE CUIDADORES DE INDIVÍDUOS HEMIPARÉTICOS
}

Vanessa Niens Van Den Broek, Fabiana Araujo Silva, Lucia Martins Barbatto, Augusto Cesinando de Carvalho

Universidade Estadual Paulista - UNESP, Faculdade de Ciências e Tecnologia FCT, Departamento de Fisioterapia, Presidente Prudente, SP. E-mail: van broek 94@hotmail.com

\section{RESUMO}

O acidente vascular encefálico é um problema de saúde mundial que traz como principal sequela a hemiparesia, sendo um dos principais causadores de incapacidade funcional e estresse para o cuidador que terá que lidar com as necessidades do paciente. $O$ objetivo deste estudo foi avaliar o nível de estresse presente nos cuidadores de indivíduos hemiparéticos, e correlacionar com as habilidades funcionais dos hemiparéticos. A metodologia consistiu em um estudo clínico com 7 cuidadores e 7 indivíduos hemiparéticos submetidos à testes que avaliam a capacidade física funcional e qualidade de vida relacionada a saúde. Nos resultados vemos que a escala QVEAVE revelou um valor médio de $193,85 \pm 21,45$ pontos. No TUG, foi obtido um tempo médio de $21,15 \pm 14,48$ segundos. A Escala Burden Interview demonstrou um valor médio de 14,28 $\pm 8,05$ pontos. Concluímos que os cuidadores apresentam baixo nível de sobrecarga emocional devido ao bom nível de qualidade de vida e mobilidade dos hemiparéticos.

Palavras-chave: Cuidador, Hemiparéticos, Sobrecarga Emocional e Qualidade de Vida.

\section{EVALUATION OF EMOTIONAL OVERLOAD OF HEMIPARETIC INDIVIDUALS CAREGIVERS}

\section{ABSTRACT}

Stroke is a global health issue that brings as main sequel hemiparesis, being one of the main causes of disability and stress for the caregiver who will have to handle the patient needs. The aim of this study was to evaluate the level of stress present in the caregivers of hemiparetic individuals, and correlate with the functional skills of hemiparetic individuals. The methodology consisted in a clinical study with 7 caregivers and 7 hemiparetic individuals submitted to tests that evaluate the functional physical capacity and quality of life related to health. In the results we see that the QVEAVE scale revealed an average value of $193.85 \pm 21.45$ Points. In the TUG, was obtained an average rate of $21.15 \pm 14.48$ Seconds. The Burden Interview Scale showed an avarage value of $14.28 \pm 8.05$ points. We concluded that caregivers have low level of emotional overload due to the good level of quality of life and mobility of hemiparetic Key-words: Caregiver, Hemiparetic, Emotional Overload, Quality of Life. 


\section{INTRODUÇÃO}

O Acidente Vascular Encefálico (AVE) é uma das principais causas de morte e incapacidades físicas no mundo. Anualmente cerca de 16 milhões de pessoas são acometidas por essa doença cerebrovascular. Devido ao acometimento físico causado pelo AVE, um impacto social e econômico acaba sendo gerado tantos nos indivíduos acometidos quanto nos cuidadores ${ }^{(1)}$.

O AVE é um problema de saúde mundial que tem como principal seqüela a hemiparesia, caracterizada por espasticidade, deficiência motora e fraqueza muscular no hemicorpo contralateral à lesão, sendo um dos principais causadores de incapacidade funcional ${ }^{(2)}$. Muitas pessoas após sofrerem um AVE passam a ter um estilo de vida sedentário tornando-se fisicamente inativas com baixo nível de aptidão física, além das alterações sensitivas, mentais, perceptivas e de linguagem ${ }^{(3)}$.

Dos indivíduos que recebem reabilitação após um AVE, quando recebem alta, 60 a 70\% deles possuem a capacidade de deambular, porém apenas $7 \%$ são capazes de caminhar em meio à outro indivíduos e em lugares com maior movimentação. Em relação à função física, apenas $14 \%$ recuperam-se totalmente e de 25 a $50 \%$ se tornam dependentes para realização das atividades de vida diária ${ }^{(4)}$.

Após sofrerem o AVE, $90 \%$ dos casos desenvolvem deficiências que os tornam mais dependentes ${ }^{(5)}$. Esta doença crônica é um agente estressor, para o cuidador que terá que lidar com a dependência e necessidades do doente ${ }^{(6)}$.

Ainda existem poucos estudos no Brasil que analisam a qualidade de vida dos indivíduos com AVE e de seus respectivos cuidadores. Devido às adaptações realizadas pelos familiares para lidar com as dependências de pacientes crônicos, há uma diminuição da qualidade de vida e uma sobrecarga dos cuidadores ${ }^{(5)}$. Vêm se tornando mais freqüente a quantidade de familiares que se colocam no papel de cuidadores de pacientes crônicos, assim como aqueles acometidos pelo AVE. O papel que esses cuidadores desempenham é fundamental para designar os melhores resultados em um tratamento, formando assim uma relação proporcional entre as habilidades do cuidador e a qualidade da reabilitação. Assim como um cuidador habilitado pode gerar um melhor grau de recuperação, a falta de informações fornecidas a ele pode ser crucial ao tratamento ${ }^{(7)}$.

O papel do cuidador é extremamente importante e é um difícil compromisso, que a maioria de nós não está totalmente preparados para empreender. Quando alguém passa por uma doença grave, como um AVE, há muito cuidados necessários além das atividades estritamente médicas. Ser um cuidador envolve uma gama de atividades, em grande parte para o preenchimento de todos os cuidados que alguém deve fazer, como a administração de medicamentos, proporcionando suporte básico na alimentação, higiene, autos cuidados e transporte ${ }^{(8)}$.

Geralmente a sobrecarga de cuidar de um paciente crônico afeta um único integrante da família, sendo este considerado o cuidador principal. Este tem que ser capaz de suportar com as dificuldades apresentadas pelo paciente como auxiliar ou realizar por completo as atividades de vida dária ${ }^{(6)}$. Muitos cuidadores são capazes de se ajustar à sua nova situação, no entanto, parte deles apresentam níveis clinicamente relevantes de estresse que pode exigir alguma forma de apoio $^{(9)}$.

O estudo tem como objetivo primário avaliar o nível de estresse presente nos cuidadores de indivíduos hemiparéticos, e como objetivo secundário correlacionar esses resultados com as habilidades funcionais dos hemiparéticos.

\section{METODOLOGIA}

Participaram deste estudo clínico, cuidadores e indivíduos hemiparéticos que estão em tratamento de fisioterapia em grupo no formato de circuito de treinamento (FGCT) no Setor de Neurologia do Centro de Atendimento de Fisioterapia e Reabilitação (CEAFIR) da Universidade 
Estadual Paulista (UNESP), campus de Presidente Prudente. Ao aceitarem participar do estudo os indivíduos e seus cuidadores foram informados sobre os objetivos e os procedimentos a serem adotados. Todos os voluntários foram informados sobre os objetivos e procedimentos do estudo e após concordarem com sua participação assinaram o termo de consentimento livre e esclarecido aceito pelo Comitê de Ética da FCT sobre o CAAE: 56717916.8.0000.5402.

Os critérios de inclusão do estudo foram: paciente com hemiparesia unilateral e encaminhamento médico com tempo de lesão $\geq$ há 12 meses; capaz de realizar a marcha com ou sem auxílio de órtese, apresente alteração de tônus de grupos musculares do membro inferior parético, identificada por escores diferentes de zero na escala modificada de Ashworth.

Os critérios de exclusão do estudo foram: dupla hemiparesia, tempo de lesão inferior a 12 meses, afasia sensitiva ou condições de saúde adversas tais como outras doenças neurológicas ou ortopédicas não relacionadas ao AVE.

Os pacientes que estiverem em tratamento de FGCT foram submetidos à testes que avaliam a capacidade física funcional e qualidade de vida relacionada a saúde, sendo estes: Time Up and Go (TUG) ${ }^{(10)}$ e Escala de Qualidade de Vida Específica para AVE (QVEAVE) ${ }^{(11)}$. Estes testes serão utilizados para comparar o nível de sobrecarga que estes pacientes determinam em seus cuidadores. Os cuidadores serão submetidos à Escala Burden Interview ${ }^{(12)}$ que é um questionário que avalia a sobrecarga emocional de pessoas que cuidam de hemiparéticos.

A FGCT é uma forma de terapia a qual utiliza exercícios, atividades e tarefas ativas funcionais específicas realizadas numa série de estações dispostas em um circuito dirigido em 10 áreas diferentes. Serão utilizados bastões, steps, halteres, caneleiras, escada progressiva, rampa, barras paralelas, garrafas, elásticos, cones, entre outros objetos. As estações são: Estação 1: Uma cadeira é disposta encostada na parede, o paciente senta-se em posição confortável e segura um haltere de $1 \mathrm{~kg}$ nas mãos, então são realizados exercícios de flexão e extensão do ombro e rotação direita e esquerda do tronco. Estação 2: são colocados 2 alvos na parede, a 1,5 metros do chão e 1 metro de distância de um alvo para o outro. O paciente segura um bastão de $1 \mathrm{~kg}$, com as duas mãos, fica em pé, de costas para os alvos, para promover uma maior rotação de tronco e flexão de ombro e tenta atingi-los com o bastão. Estação 3: o corrimão é utilizado como suporte em que é amarrada uma corda elástica de calibre médio a 60 centímetros do chão. Realiza-se o movimento de flexão resistida de ombro até o nível da mesma articulação. Os cotovelos permanecem estendidos durante a realização do exercício. Estação 4: um step é colocado no chão e o paciente realiza o movimento de agachamento, flexão de joelho e retorno à posição inicial, em cima do step, segurando no corrimão como auxílio. Nas 8 últimas semanas o step será substituído pelo bozu. Estação 5: um cone é colocado a uma distância de 5 metros de uma cadeira. 0 indivíduo deve partir da posição sentada, marchar e dar a volta no cone e em seguida voltar e sentar-se na cadeira. O movimento deve repetir-se até segunda ordem. Estação 6: para a realização dessa tarefa é utilizado um armário e algumas garrafas de $1 \mathrm{~kg}$ cada. As garrafas ficam dispostas em um nível baixo do armário e é solicitado ao paciente que transfira todas as garrafas ao nível mais alto que ele conseguir, em seguida o mesmo deve voltar as garrafas ao nível mais baixo. Estação 7: utilizando 3 ciclos ergômetros, o paciente realiza movimento circular com a articulação glenoumeral, tais movimentos podem ser realizados tanto no sentido horário quanto no antihorário. Estação 8: é realizada nas barras paralelas, em que o paciente deve ultrapassar obstáculos de $5 \mathrm{~cm}$ de altura inicialmente, realizando marcha lateral. Assim que o indivíduo chega ao final da barra, deve voltar ao ponto de partida e assim sucessivamente. Nas 8 últimas semanas, os obstáculos passarão a ter uma altura de 7,5 centímetros. Estação 9: o paciente realiza marcha e/ou marcha reversa na escada com rampa. Utiliza-se em cada perna, uma caneleira de $0,5 \mathrm{~kg}$. Nas últimas semanas o peso será dobrado. Estação 10: são colocados 3 steps no chão, de forma que fiquem em contato com a parede. O paciente deve realizar movimento de subida e decida, pelo tempo de 3 minutos. É realizado movimento de flexão, extensão de quadril, joelho e tornozelo. 
Os dados coletados foram alocados em planilhas e para o tratamento estatístico foi utilizado o teste de Shapiro-Wilk para testar a normalidade do conjunto de dados e, com base nos parâmetros fornecidos, foi realizada a análise descritiva e os dados foram apresentados em valores de média e desvio-padrão.

\section{RESULTADOS}

O estudo foi composto por 7 pacientes com hemiparesia sendo 3 mulheres e 4 homens com média de idade $62,71 \pm 8,53$ anos e por 7 cuidadores com média de idade de 53,71 $\pm 14,39$ ano com diferentes graus de parentesco. A escala QVEAVE revelou um valor médio de 193,85 $\pm 21,45$ pontos. Os valores de referência da escala QVEAVE variam de 49 - 245 pontos sendo que quanto maior a pontuação melhor a qualidade de vida e, portanto a população estudada apresenta um bom nível da qualidade de vida. No teste de mobilidade TUG, foi obtido um tempo médio de teste de $21,15 \pm 14,48$ segundos sendo que quanto maior o tempo gasto para realização do teste menor a mobilidade apresentada pelo paciente. A Escala Burden Interview demonstrou um valor médio de $14,28 \pm 8,05$ pontos revelando uma baixa sobrecarga emocional dos cuidadores, pois, os valores de referência desta escala variam de 0 - 88 pontos, sendo que quanto menor a pontuação menor a sobrecarga do cuidador. Os valores médios dos resultados estão representados na figura 1.

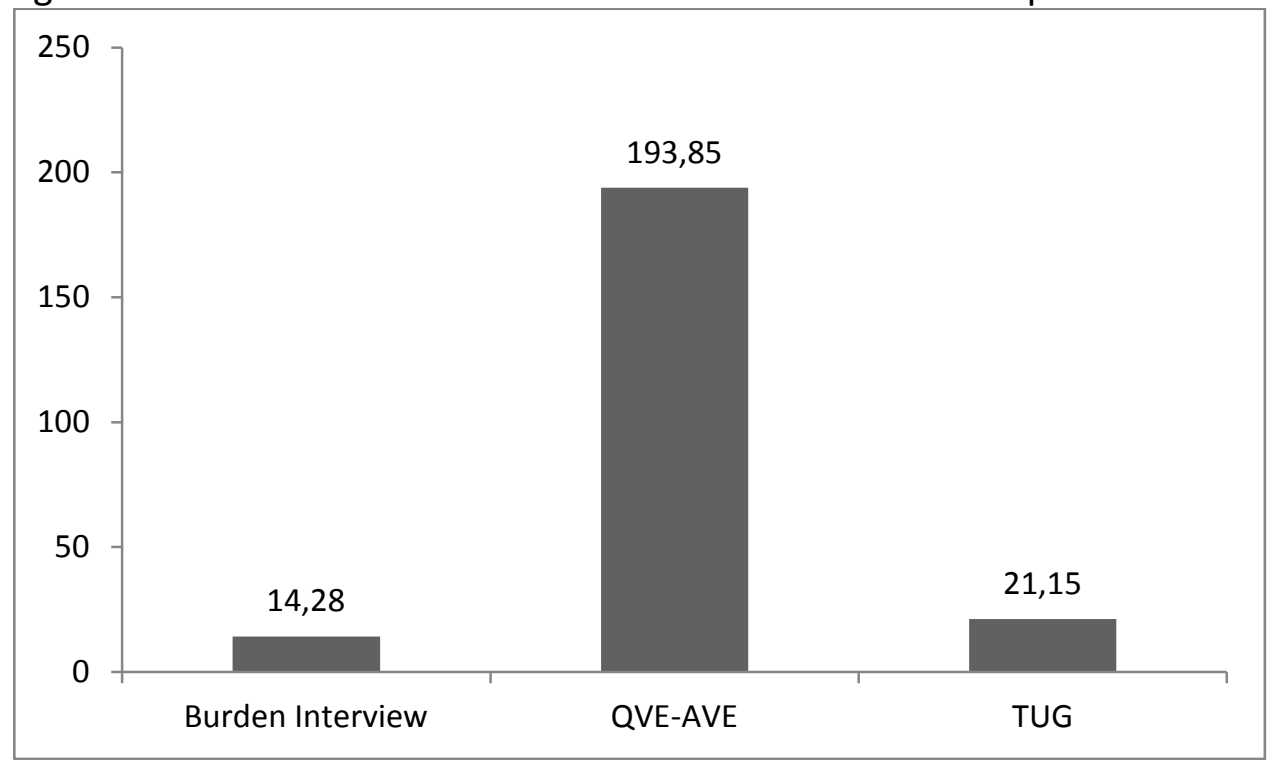

Figura1.

\section{DISCUSSÃO}

As dependências geradas nos indivíduos hemiparéticos fazem com que os cuidadores apresentem uma sobrecarga emocional devido à mudança que deve ser realizada em suas vidas para se adaptarem às necessidades dos mesmos. Os resultados do nosso estudo demonstraram que os cuidadores não apresentam índices altos de sobrecarga, pois os pacientes avaliados não apresentam baixo nível de qualidade de vida ${ }^{(5)}$.

Hemiparéticos possuem dificuldades de deambular na comunidade devido ao risco de quedas necessitando da ajuda de seus cuidadores, gerando assim aumento da sobrecarga do mesmo, porém nesse estudo verificamos que os pacientes apresentaram mobilidade suficiente adequada para deambularem na comunidade e isto pode ter sido o fator determinante na qualidade de vida destes pacientes e no nível de estresse dos cuidadores ${ }^{(4)}$.

A existência de um indivíduo com sequelas limitantes como aquelas encontradas pós-AVE requer uma reorganização das tarefas no ambiente doméstico designadas aos seus familiares. Devido essa remodelação de papéis, a família sofre alterações à nível psíquico, físico, econômico e social ${ }^{(13)}$. Devido à remodelação dos papéis, é gerada uma sobrecarga no cuidador, sendo esta 
definida como uma perturbação que ocorre pela necessidade de lidar com um indivíduo com incapacidades físicas e mentais ${ }^{(14)}$. Pacientes hemiparéticos que possuem uma baixa qualidade de vida e baixa mobilidade determina um aumento da sobrecarga emocional nos cuidadores, porém no presente estudo, os dados encontrados nos mostram uma boa qualidade de vida dos pacientes hemiparéticos e uma baixa sobrecarga dos cuidadores ${ }^{(15)}$.

Podemos inferir através deste estudo que o paciente com a maior qualidade de vida gera também uma menor sobrecarga emocional em seu cuidado, todavia deve-se ressaltar que o tamanho da amostra utilizado no presente estudo foi pequeno e nenhum dos pacientes avaliados apresentou alto grau de comprometimento na marcha determinado pelo TUG.

\section{CONCLUSÃO}

Os cuidadores desta pesquisa apresentam baixo nível de sobrecarga emocional devido ao bom nível de qualidade de vida e mobilidade dos hemiparéticos.

\section{REFERÊNCIAS}

www.brasil.gov.br/saude/2012/04/acidente-vascular-cerebral-avc- Acessado em 24/04/2016 às $14 \mathrm{~h} 25 \mathrm{~min}$.

Dobkin BH. Clinical practice. Rehabilitation after stroke. N Engl J Med. 2005 Apr 21;352(16):167784. https://doi.org/10.1056/NEJMcp043511.

Braun, A.; Herber, V.; Michaelsen, S.M. Relação entre nível de atividade física, equilíbrio e qualidade de vida em indivíduos com hemiparesia. Rev Bras Med Esporte, vol.18 no.1 São Paulo Jan./Feb. 2012

DONOVAN, K.; LORD, S. E.; MCNAUGHTON, H. K.; WEATHERALL, M. Mobility beyond the clinic: the effect of environment on gait and its measurement in community-ambulant stroke survivors. Clinical Rehabilitation, v. 22, n. 6, p. 556-563, 2008. https://doi.org/10.1177/0269215507085378

LIMA, Mary Lícia de et al. Qualidade de vida de indivíduos com acidente vascular encefálico e de seus cuidadores de um município do Triângulo Mineiro. Revista Brasileira Epidemiol, Uberaba, p.453-464, jun. 2014

FONSECA, Natália da Rosa; PENNA, Aline Fonseca Gueudeville. Perfil do cuidador familiar do paciente com seqüela de acidente vascular encefálico. Ciência \& Saúde Coletiva, Salvador, v. 4, n. 13, p.1175-1180, 2008.

GARANHANI, Márcia Regina et al. A experiência de pacientes e cuidadores após acidente vascular encefálico: uma revisão narrativa da literatura. Ciencia Cuidado e Saude, Londrina, v. 2, n. 8, p.242-249, 2009

MOON, Marilyn. The Unprepared Caregiver. The Gerontologist, Maryland, v. 00, n. 00, p.1-6, 21 abr. 2016. Oxford University Press (OUP). http://dx.doi.org/10.1093/geront/gnw080.

KRUITHOF, Willeke J. et al. Caregiver burden and emotional problems in partners of stroke patients at two months and one year post-stroke: Determinants and prediction. Patient Education And Counseling, Amsterdam, p.1-9, 08 abr. 2016. Elsevier BV.

http://dx.doi.org/10.1016/i.pec.2016.04.007. 
Podsiadlo D, Richardson S. The timed "Up \& Go": a test of basic functional mobility for frail elderly persons. J Am Geriatr Soc. 1991 Feb;39(2):142-8. https://doi.org/10.1111/j.15325415.1991.tb01616.x

NETO MG. Aplicação da Escala de Qualidade de Vida Específica para AVE (EQVE-AVE) em Hemiplégicos Agudos: Propriedades Psicométricas e sua Correlação com a Classificação Internacional de Funcionalidade Incapacidade e Saúde. [Dissertação]. Belo Horizonte: UNIVERSIDADE FEDERAL DE MINAS GERAIS;2007.

SCAZUFCA, Marcia. Brazilian version of the Burden Interview scale for the assessment of burden of care in carers of people with mental illnesses. Rev Bras Psiquiat, São Paulo, v. 1, n. 24, p.12-17, 2002.

Ferreira et al. Validação da escala de Zarit: sobrecarga do cuidador em cuidados paliativos domiciliários, para população portuguesa. Cadernos de Saúde, Vol. 3 N. 2 - pp. 13-19. 2015.

Santos P. O familiar Cuidador em ambiente domiciliário: Sobrecarga física, emocional e social. Tese de Mestrado em saúde pública. Lisboa: Escola Nacional de Saúde Pública/Universidade Nova de Lisboa; 2005

Sousa AG, Zarameli RC, Ferrari RAM, Frigero M. Avaliação da qualidade de vida de cuidadores de pacientes com seqüelas neurológicas. ConScientiae Saúde. 2008 [2016 ago08];7(4):497-502. Disponível em: http://www.redalyc.org/articulo.oa?id=92911724012. 